\title{
Research on the Human Rights and Cultural Protection of Environmentally Displaced Persons under Rising Sea Levels
}

\author{
Rui Xie $\mathbb{D}^{1},{ }^{1}$ Wen-Bo Li $\left(\mathbb{D},{ }^{2}\right.$ Meng-Chun Lin $\mathbb{D}^{1},{ }^{1}$ Di Lu $\left(\mathbb{D},{ }^{3}\right.$ and Jia-Ming Zhu $\mathbb{D}^{3}$ \\ ${ }^{1}$ School of Accounting, Anhui University of Finance and Economics, Bengbu 233030, China \\ ${ }^{2}$ School of Finance, Anhui University of Finance and Economics, Bengbu 233030, China \\ ${ }^{3}$ School of Statistics and Applied Mathematics, Anhui University of Finance and Economics, Bengbu 233030, China
}

Correspondence should be addressed to Jia-Ming Zhu; zhujm1973@163.com

Received 8 December 2020; Revised 1 January 2021; Accepted 15 January 2021; Published 27 January 2021

Academic Editor: M. Irfan Uddin

Copyright ( $) 2021$ Rui Xie et al. This is an open access article distributed under the Creative Commons Attribution License, which permits unrestricted use, distribution, and reproduction in any medium, provided the original work is properly cited.

\begin{abstract}
In recent years, due to factors such as rising sea levels, several island nations such as Maldives, Tuvalu, Kiribati, and the Marshall Islands are in danger of disappearing completely. When the land of an island country disappeared, the human rights protection of Environmentally Displaced Persons in the migration process and the possible loss of their unique culture, language, and lifestyle have aroused great concern. We call such Environmentally Displaced Persons as EDPs. This study selects the EDPs' data of 241 countries or regions from 2008 to 2018, establishes an ARIMA model, and predicts the future population of EDPs. By combining the influencing factors of cultural loss, the risk assessment model of cultural loss is established to evaluate the possibility of cultural loss during the migration process of EDPs. We have established a Bayesian Network and a Fault Tree Model to demonstrate the improvement brought about by the implementation of policy recommendations from both qualitative and quantitative perspectives and use the method of fault tree analysis to illustrate the importance of policies from the degree of probability reduction after policy changes. Finally, based on the above model establishment and data analysis, corresponding countermeasures are proposed to protect EDPs' human rights from being violated and their culture will not be lost.
\end{abstract}

\section{Introduction}

In the general context of global climate change, the likelihood of climate risks such as global warming and melting glaciers and extreme sudden catastrophic events has increased. These catastrophic events will put several island nations such as the Maldives, Tuvalu, Kiribati, and the Marshall Islands in danger of disappearing completely due to rising sea levels. When the land of those island nations disappeared, the island's population had to leave their homes and choose to migrate in order to survive. In the process of migration, they not only face the danger of life but also the risk of losing their unique culture, language, and lifestyle $[1,2]$. The actual or potential large-scale climate migration caused by sea level rise has become a serious challenge facing human society in the 21 st century.

Based on the existing research literature, it is found that since the Industrial Revolution, climate change has led to increased climate risk, frequent occurrences of climate disasters, and environmental changes, resulting in an increase in the number of climate immigrants [3]. This requires us to use relevant mathematical models to predict the number of EDPs in the future. Temporary migration is constantly evolving into permanent immigration, and various factors are intertwined. The task of making relevant policy recommendations to protect the human rights and culture of EDPs is imminent $[4,5]$.

\section{Data Sources and Assumptions}

The data in this paper come from Problem F of the 2020 American College Students Mathematical Contest in Modeling. In order to facilitate the research of the problem, the following hypotheses are proposed: (i) No other disasters such as natural disasters and war diseases have increased the number of EDPs. (ii) There is no incident of malicious false report information, so the data provided are accurate and reliable. (iii) There was no large-scale migration of residents 
before EDPs' immigration. (iv) The risk of cultural loss of EDPs can be qualitatively distinguished by grade. (v) The receiving country has similar natural conditions to the original country, and the number of people living will not be affected by environmental differences. (vi) High-risk areas with rising sea levels will have little cultural damage caused by other factors before reaching the warning line.

\section{Forecast of the Number of EDPs}

3.1. Predictive Analysis of the Time Series Model. Time series means a series of observations obtained in chronological order during production and scientific research. They are random data formed by one variable or multiple variables at different times, which can reflect the development and change law of the observed phenomenon [6]. The time series model is to use a mathematical model to fit the time series of the target object and to analogize and extend according to its direction and trend, predicting the level that the object can reach in the next period.

Most small island states come from developing countries that are small, densely populated, have limited financial resources, and are less resilient to natural hazards, and some researchers have found a linear rise in sea level of $3.44 \mathrm{~mm}$ per year. The relevant data are shown in Figure 1.

According to the statistics, as of the end of 2016, there were 65.6 million forced displacements worldwide, of which 22.5 million were refugee status, 40.3 million were internally displaced, and 10 million were stateless or at risk of statelessness. There were 10.3 million new displaced persons in 2016, including 6.9 million new internally displaced persons and 3.4 million new refugees and asylum seekers. There were 552,200 refugees returning home in 2016, and 189,300 refugees were resettled, accounting for only $2.45 \%$ and $0.84 \%$ of the total refugees, respectively, which are still at a relatively low proportion. The data as of 2016 show that the number of new forced displaced persons in 2016 was 300,000 , compared with 5.8 million in 2015 and 8.3 million in 2014, a slowdown in growth.

Environmentally Displaced Persons (EDPs) are closely related to regional and national natural environments. Therefore, the research, prediction, and control of EDPs are related to the national economy and people's livelihood. We selected refugee population data from 241 countries and regions from 2008 to 2018 and used time series analysis methods to build models for analysis and prediction. The relevant situation is shown in Table 1.

3.2. ARIMA Model Prediction. The ARIMA model is a widely used model in time series analysis. Its structure is as follows:

$$
\left\{\begin{array}{l}
\Phi(B) \nabla^{d} x_{t}=\Theta(B) \varepsilon_{t}, \\
E\left(\varepsilon_{t}\right)=0, \operatorname{Var}\left(\varepsilon_{t}\right)=\sigma_{\varepsilon}^{2}, E\left(\varepsilon_{t} \varepsilon_{s}\right)=0, s \neq t \\
E\left(x_{s} \varepsilon_{t}\right)=0, \quad \forall s<t
\end{array}\right.
$$

where $\quad \Delta^{d}=(1-B)^{d}$ and $\Phi(B)=1-\phi_{1} B-\phi_{2} B^{2}-\cdots-\phi_{p} B^{p}$ is the autoregressive coefficient polynomial. The basic idea of ARIMA model modeling is to combine the difference operation with the ARIMA model [7-9]. First, the nonstationary time series is converted to a stationary time series by a certain order of difference, and then the ARIMA model is fitted to the difference sequence. Its operation process is shown in Figure 2.

3.3. Fit of the Model. We use the software SPSS to process and analyze the above data to obtain the model fitting degree. From the following table, we can see that the model has a good fitting effect. The fitted results are shown in Table 2.

3.4. Predicted Result. We use the moving average method for predictive analysis of the data and find that the growth rate of EDPs has slowed in recent years. Considering natural disasters, war, and other factors, it is predicted that the number of people in the next five years will reach 300 million. The predicted result is shown in Figure 3.

\section{Risk Assessment of EDPs' Culture Loss}

Migration is one of the ways in which humans respond to climate change and sustain themselves. But when many refugees leave their homes and move to other countries, how should the language and culture of these climate refugees be protected from loss?

As we all know, there are many risks of cultural loss. In the process of normal cultural exchange, cultural assimilation cannot be avoided. In the process of large-scale EDPs' immigration, cultures collide on a large scale, and the weaker side of the culture will gradually introduce and learn the strong culture [10-12]. In this process, EDPs largely occupy the culturally disadvantaged side. The EDPs' culture is at risk of loss. How to protect the EDPs' culture without affecting the lives of refugees and the human rights of refugees has become a problem that needs to be solved. With the rise of many EDPs' immigrants, the risk of cultural loss has also become one. Facing the risk of cultural loss, we have established a risk assessment model to provide a basis for determining the risk of cultural loss among immigrants in order to propose governance policies.

4.1. Risk Identification of EDPs' Culture Loss. There is an old saying in China, one party supports water and soil to support one party. This sentence makes sense. EDPs were forced to emigrate to another country or region due to climate change and lost his home [13]. This has greatly changed the living environment of immigrants. Changes in the living environment will also subtly affect people's daily life and living skills.

4.1.1. Natural Environmental Risks. A large part of the impact of climate change is that sea levels rise and islanders lose their homes. Therefore, most EDPs are island citizens or islanders. When they are forced to migrate, the living environment will change and the migrants will live in the 


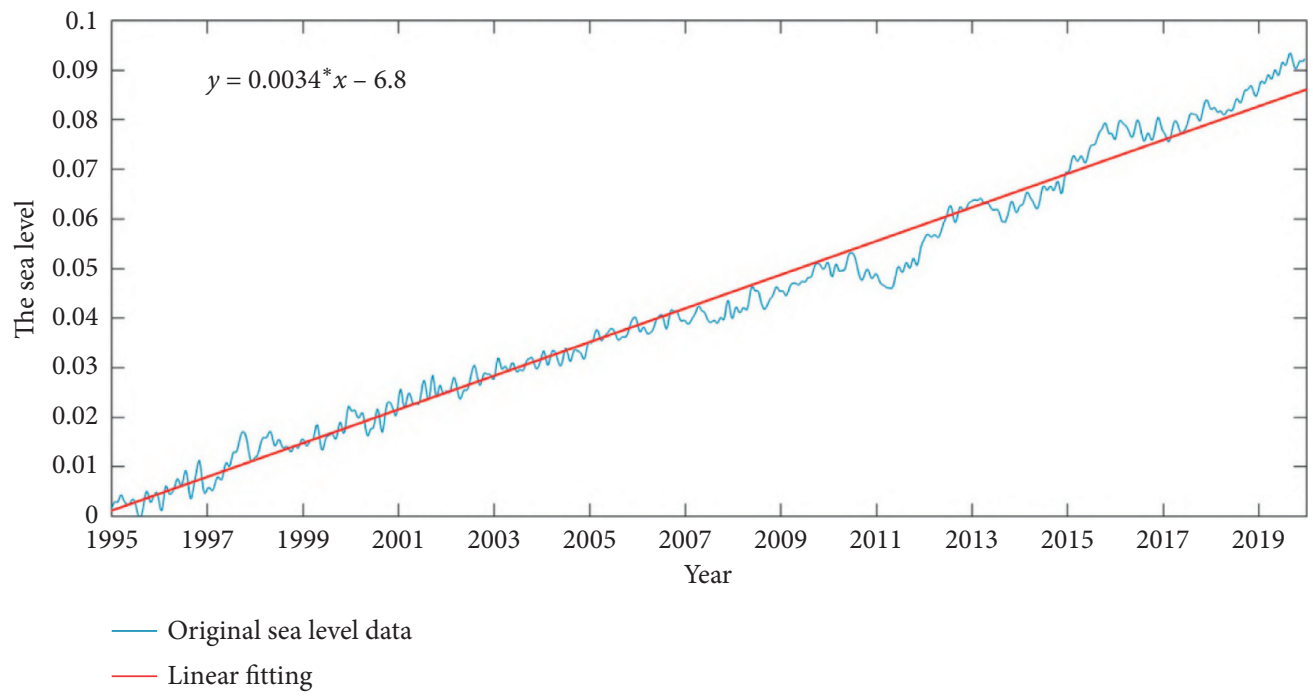

Figure 1: The original sea level data and its linear fitting.

TABle 1: Refugee population data in 241 countries and regions from 2008 to 2018.

\begin{tabular}{lcccc}
\hline Model & $\begin{array}{c}\text { Model fitting statistics } \\
\text { Stationary } r \text {-square }\end{array}$ & \multicolumn{2}{c}{ Ljung-Box Q (18) } \\
Statistics & DF \\
\hline 2008-model_1 & 0.789 & 14.270 & 17 & 17 \\
2009-model_2 & 0.376 & 16.484 & 17 & 0.648 \\
2010-model_3 & 0.431 & 15.151 & 17 & 0.490 \\
2011-model_4 & 0.799 & 15.109 & 17 & 0.585 \\
2012-model_5 & 0.799 & 16.151 & 17 & 0.588 \\
2013-model_6 & 0.799 & 16.152 & 17 & 0.513 \\
2014-model_7 & 0.421 & 17.297 & 0.434 \\
2015-model_8 & 0.798 & 18.873 & 17 & 0.336 \\
2016-model_9 & 0.799 & 19.041 & 17 & 0.326 \\
2017-model_10 & 0.341 & 22.924 & 17 & 0.152 \\
2018-model_11 & 0.418 & 19.387 & & 0.307 \\
\hline
\end{tabular}

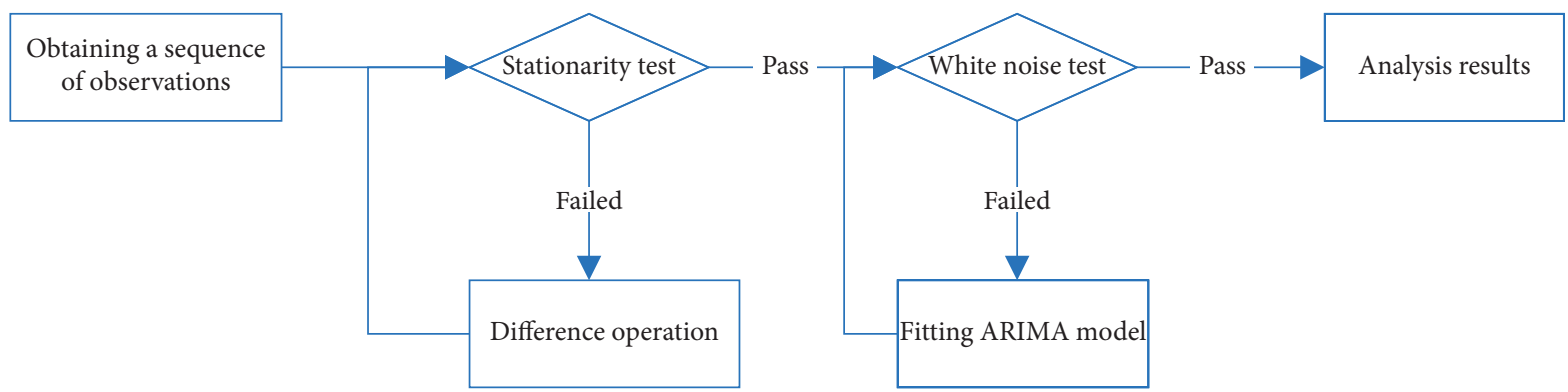

FIGURE 2: Operation process of ARIMA model.

TABLe 2: Fitted results.

\begin{tabular}{lcccc}
\hline Fitting statistics & Mean & SE & Minimum value & Max \\
\hline Stationary $r$-square & 0.615 & 0.210 & 0.341 & -0.002 \\
$r$-square & -0.004 & 0.002 & -0.007 & $3,744,840.574$ \\
RMSE & $2,465,750.605$ & $763,411.412$ & $1,791,940.273$ & $4,375,667.883$ \\
MAPE & $2,826,566.868$ & $1,006,762.033$ & $1,177,054.190$ & $116,236,572.702$ \\
Max APE & $76,277,721.816$ & $26,943,962.281$ & $22,436,386.550$ & $1,838,839.228$ \\
MAE & $1,216,284.594$ & $365,357.622$ & $865,783.959$ & $24,910,490.710$ \\
Max AE & $18,142,786.772$ & $4,053,282.125$ & $14,539,257.225$ & 30.296 \\
Normalized BIC & 29.377 & 0.590 & 28.821 & \\
\hline
\end{tabular}




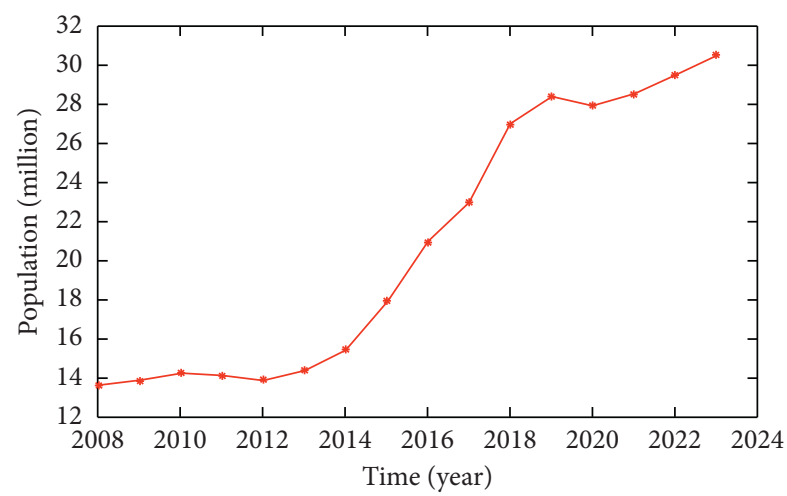

Figure 3: Predicted result.

islands. The life skills and habits used will change as a result $[14,15]$. For example, people who often live on islands have a unique fishing skill, but residents who migrate to land no longer need this life skill. Over time, this unique catch fish culture is lost in the lives of immigrants.

4.1.2. Social and Environmental Risks. After immigrants lost their homes, they also lost their social networks. Postimmigrant refugees entered a new social environment after immigration. Immigrant refugees must face language differences, religious beliefs, and differences in social network structures. This new social environment has played a certain role in promoting cultural exchanges, and it is also slowly affecting the inheritance and development of foreign cultures [16].

4.1.3. Other Risks. There are some other risks in the process of EDPs large-scale immigration. For example, different immigration methods can lead to different degrees of cultural loss. Centralized immigration is easier than decentralized immigrants' cultural retention. Immigration destinations also have an impact on cultural loss. Cultural preservation is better served by moving to an environment like the one in which EDPs lived [17]. On the contrary, the wrong choice of immigration destination will also be a blow to culture.

4.2. Calculation of Evaluation Index Based on Analytic Hierarchy Process. An important part of the analytic hierarchy process is to construct a judgment matrix. When determining the weights between factors at each level, compare the two factors and use the 1-9 scale method to negative them. Get the evaluation index weights layer by layer. The result of sorting results in the judgment matrix $P$ :

$$
P=\left(\begin{array}{ccc}
p_{11} & \cdots & p_{1 n} \\
\vdots & \ddots & \vdots \\
p_{n 1} & \cdots & p_{n n}
\end{array}\right),
$$

where $n$ is the number of evaluation indicators. For the judgment matrix $P$, a square root method is used to solve the eigenvectors; that is, the product $M_{i}$ of each row element in
$P$ is calculated and the nth root of $M_{i}$ is obtained. The specific calculation formula is as follows:

$$
M_{i}=\prod_{j=1}^{n} P_{i j}, \bar{\omega}_{i}=\sqrt[n]{M_{i}}, W_{i}^{\prime}=\frac{\overline{\omega_{i}}}{\sum_{i=1}^{n} \bar{\omega}_{i}} .
$$

Perform consistency check to ensure the accuracy of the judgment matrix, where the consistency is calculated using the index $C_{i}$. The smaller the value of $C_{i}$, the greater the consistency of the proof. The definition of the consistency index is

$$
C_{i}=\frac{\lambda-n}{n-1} .
$$

When $C_{i}=0$, there is complete consistency. $C_{i}$ is close to 0 , there is satisfactory consistency, and the larger $C_{i}$, the more serious the inconsistency.

\subsection{Fuzzy Comprehensive Evaluation Model}

4.3.1. Establish a Risk Set for Cultural Loss. The risk of losing immigrant culture can be divided into many types. For example, the mother tongue gradually loses its first language status after immigration. The conflict between the original customs and the customs of the immigrant-receiving country can be gradually reduced, and the living habits will also change after changing the living environment.

According to the risk analysis and identification, in the process of EDPs' immigration, the set of risk factors for cultural loss is expressed as

$$
U=\left\{u_{1}, u_{2}, \ldots, u_{n}\right\} .
$$

4.3.2. Find the Factors that Cause the Risk of Cultural Loss. The development of EDPs' immigration culture is affected by many objective conditions, such as migration methods, number of immigrants, and immigration destinations, and other factors will have different degrees of impact on the development of immigration culture. We set the cause of cultural loss as

$$
Y=\left\{y_{1}, y_{2}, \ldots, y_{p}\right\} .
$$

4.3.3. Select the Risk Assessment Set. We divide the risk of cultural loss into 5 levels to arrive at the evaluation set:

$$
V=\left\{v_{1}, v_{2}, v_{3}, v_{4}, v_{5}\right\} .
$$

Set (7) represents the existence of immigration culture, which means total loss, basic loss, partial reservation, basic reservation, and full reservation. We set total loss equal to 1.0, basic loss equal to 0.75 , partial reservation equal to 0.5 , basic reservation equal to 0.25 , and complete reservation equal to 0 .

4.3.4. Determine the Weight of Each Factor. Use the analytic hierarchy process to decompose the risk of cultural loss into a hierarchical structure, and then analyze it layer by layer to construct a judgment matrix for each risk factor and 
subfactor, calculate the eigenvalues, and check consistency to obtain the weight of each risk factor and subfactor. The factor weight set is expressed as

$$
W=\left\{w_{1}, w_{2}, \ldots, w_{m}\right\}
$$

According to the characteristics of the risk of immigration culture loss, the Delphi method is used to determine the weight of each indicator. According to the risk factors in each layer $u_{i}$ relative to the importance of the total risk $u$, each risk factor is given a corresponding weight and the number of weights at each level. $w_{i}$ should meet the conditions of normality and nonnegativity. It is expressed as

$$
\sum_{i=1}^{m} w_{i}=1, w_{i} \geq 0 .
$$

4.3.5. Establish Membership and Evaluation Matrix. Perform a relative rating of each of the risk factor indicators of cultural loss in the cultural loss risk set $u$ to establish an evaluation set $R$ :

$$
R=\left(\begin{array}{ccc}
r_{11} & K & r_{1 n} \\
M & O & M \\
r_{m 1} & L & r_{m n}
\end{array}\right)
$$

Among them, $r$ is the membership degree of the subfactor layer index $u_{k i}$ for the $i$-th level evaluation $v_{j}$, that is,

$$
r=\frac{v}{\sum_{j=1}^{n} v_{i j}}, \quad i=1,2, \ldots m, j=1,2, \ldots, n .
$$

4.3.6. Make Multilevel Fuzzy Comprehensive Evaluation. Multiply the indicator weight set with the evaluation matrix to get the final evaluation result, that is, various risks and total risks:

$$
E_{i}=w_{i} \times r_{i} \times V^{\prime} .
$$

Among them, $V^{\prime}$ is the transposed vector of the row vector $V$. Compare the risk evaluation result with the evaluation set $V$ to get the identification risk magnitude and provide a decision-making basis for the protection of EDPs' immigration culture.

\section{Management Policy for EDPs' Migration}

5.1. Clarify the Coverage of EDPs. The clarification and standardization of climate resettlement guarantee scope is an important prerequisite for effective resettlement and followup assistance. It is necessary to formulate treaties or agreements on the protection of the rights and interests of climate migrants and international assistance based on the basic spirit of equally protecting the climate rights and interests of global residents $[18,19]$.

The hundreds of years of emissions from the major responsible countries that have not yet actively adopted effective measures to reduce greenhouse gas emissions are the main causes of current environmental problems. They are obliged to take a series of effective measures to assist source countries to establish green economic growth models and efficient disaster prevention and control mechanisms and to accommodate temporary and long-term immigration based on specific conditions [20,21].

Based on the international practice of georelief, neighboring countries are also obliged to accept and assist the migration activities of the source country and to maintain as much as possible the language characteristics, traditional culture, and value system of immigrant groups. There is a need to formulate a convention that clearly states that countries and international organizations around the world have the obligation to help developing countries suffering from climate disasters to improve their disaster relief capabilities and extend their residence time without endangering their own security, economic development, and management order. Plan to relocate the affected people to other places to ensure that EDPs' human rights and culture will not be lost.

5.2. Establish a Management System to Specifically Address Climate Migration. First, a set of social management and governance mechanisms that can flexibly respond to climate migration can be established in the international community, including exploring and constructing scientific and reasonable social management and governance mechanisms based on understanding and analyzing the various risks that climate migration may bring in order to enable climate migrants to better adapt to the changes in the natural and social environment caused by climate change [22].

In addition, it is necessary to strengthen the construction of disaster forecast and early warning mechanisms related to climate change, to establish historical information bases and data-sharing platforms for major climate disasters in the region, to further strengthen the construction of a climate migration assistance system, to establish an efficient and comprehensive assistance system, and to strengthen the construction of the rescue system.

Finally, UNHCR needs to strengthen immigration management and the exchange of international experience and the construction of an efficient climate migration emergency management mechanism and to promote the construction of a social and cultural adaptation mechanism to address climate migration-related issues $[23,24]$. Based on the understanding that prevention is better than governance, strengthen monitoring and evaluation of climate resettlement.

\subsection{Maintaining Concentration after EDPs' Migration.} Based on the basic theoretical framework model of the relationship between climate change and population migration, a response model of extreme climate change and disaster resettlement was established.

In order to keep the original culture of these climate refugees from disappearing with the migration, those countries passing by during migration and the countries that receive these refugees should pay attention to the problem of 
crowd distribution. According to the example of Chinatown in the United States, this is a problem that many Chinese people migrate and live in concentration. The Chinese in Chinatown also celebrate the Chinese New Year every year, keeping their original culture intact [25]. Therefore, for the cultural protection of climate refugees, the most important thing is to allow them to form a community to maintain a concentrated life.

\section{Improvement after Implementation}

6.1. Multi-Index System Function Mechanism of EDPs' Culture. According to the above analysis, the study compares the EDPs' cultural control with the economic benefits of each estimate; in addition, given the support of EDPs' policy support, the environment, and other factors, the EDPs' cultural risk control forecast is considered as a multiindex decision function, which is as follows:

$$
U=u\left(f_{1}, f_{2}, \ldots, f_{n}\right) .
$$

Among them, $f_{1}, f_{2}, \ldots, f_{n}$, respectively, represent different decision combinations that form EDPs' cultural risks, and each combination corresponds to forming an effect function; EDPs' cultural risk control decision-making is the comprehensive evaluation result of a multidecision combination, that is, the comparison result of the comprehensive decision-making economic benefits represented by the above formula (13). To simplify the combination of different risk control decision-making combinations to form a comprehensive effect function in the context of a decision group conflict, research the decision combinations corresponding to the above different utilities which are arranged according to vector groups; that is,

$$
U=k_{1} f_{1}+k_{2} f_{2}+\cdots+k_{n} f_{n} .
$$

Each decision in equation (14) as a vector group's decision factor corresponds to different EDPs' cultural risk investment elements. The corresponding real estate investment risk control decision can be arranged and combined according to different economic effects of the decision according to the vector group. After transposition, a basic matrix corresponds to the vector group; that is,

$$
A=\left[\begin{array}{cccc}
a_{11} & a_{12} & \ldots & a_{1 i} \\
a_{21} & a_{22} & & a_{2 i} \\
\vdots & \ddots & \vdots \\
a_{i 1} & a_{i 2} & \cdots & a_{i i}
\end{array}\right] .
$$

In formula (15), different vector groups correspond to different control risk decisions, and each matrix element of the matrix forms an EDPs' cultural control decision economic benefit evaluation value.

Then, according to different EDPs' cultural risk control decisions, the corresponding economic benefits are evaluated. The evaluation mainly depends on the process of minimum variance; that is, the weight of different risk decisions is determined by the cross-product ratio of each row and column element. The $j$-th input element of the $i$ - type risk control scheme is represented by the $j$-th column of the row, and then the budget of the $i i$-th row corresponds to the combination of the $i$-input elements that represent the $i$ type risk control scheme. The corresponding weight of the corresponding variance can be obtained by the following expression:

$$
P_{\min }\left\{z=\sum_{i=1}^{4} \sum_{j=1}^{4}\left(a_{i j} k_{j}-k_{i}\right)^{2}\right\} .
$$

Equation (16) represents the variance between the corresponding decision of the elements that focus on different EDPs' cultural risk control decisions and the $i$-th row and $j$-th column representing the $j$-th input element's economic effect value of the $i$-risk control scheme, and according to the impact, EDPs' cultural risk control makes one-by-one measures of different combination decisions and finally forms a mathematical summation formula.

In addition, the $i$-th row and the $j$-th column of each $i$-th investment risk control scheme meet the assumption that the economic effect value of the $j$-th input factor is 1 . The corresponding value is a relative weight, so they are all decimals. The following mathematical conditions are as follows:

$$
\sum_{j=1}^{4} k_{j}=1, \quad k_{j} \in 0,1
$$

\subsection{Probability Distribution of Bayesian Network Decision} Reasoning. According to the basic principles of Bayesian theory, research the prior and posterior probabilities based on network decision reasoning, then carry out the corresponding Bayesian probability combination according to the prior probabilities through the posterior probabilities, and then combine formulas (15) and (16). Carry out the basic probability measure of Bayesian network decision inference, from which the basic Bayesian inference model based on the prior probability and posterior probability distribution can be obtained:

$$
p\left(w_{i} \mid x\right), i=\frac{p\left(x \mid w_{i}\right)_{P}\left(w_{i}\right)}{\sum p\left(x \mid w_{i}\right) p\left(w_{i}\right)}, \quad i=1,2, \ldots, j .
$$

The distribution of this probability depends on the prior probability of the fuzzy state corresponding to the original decision corresponding to equation (18), and then the fuzzy information of the posterior probability is modified according to the EDPs' cultural risk control decision corresponding to the fuzzy information. Combined with equation (15), the corresponding matrix is modified accordingly to form an extension based on the basic Bayesian theoretical model.

Then, the economic benefits corresponding to different levels of EDPs' cultural risk control decisions are divided into two levels: the maximum and the minimum, each level corresponds to several EDPs' cultural risk control decisions, and Bayesian is obtained according to the difference between 
the mean and the maximum. The network decision reasoning indicator groups are

$$
\widehat{I}_{i j}^{r}=\frac{I_{i j}^{r}-\min _{1 \leq i<q}\left(I_{i j}^{r}\right)}{\max \left(I_{i j}^{r}\right)-\min _{1 \leq i<q}\left(I_{i j}^{r}\right)}, \quad i, j=1,2, \ldots, n .
$$

6.3. Bayesian Network Improvements. According to the above analysis, in order to avoid the difference in investment risk decisions between different decisions, firstly perform independent first-order regression according to the above equations (18) and (19), which are

$$
r s_{i t}=I_{t}+r s_{i, t-1}^{\prime} I_{i}+X_{i, k_{i}}{ }^{\prime}+X_{i t} .
$$

Among them, $r s$ is responsible for characterizing the sum of the total risk measurement indicators of the above EDPs' cultural control process, $I$ is responsible for characterizing various risk control decisions, and $X$ is responsible for characterizing the decision weight parameters facing the different decisions mentioned above, which meets a Basic vector trend characteristic, which are

$$
\left|R S_{i}\right|<1, \quad i=1,2, \ldots, N ; t=1,2, \ldots, T .
$$

Next, we study the first-order Bayesian network learning simulation for the total economic benefit corresponding to the total risk measure index corresponding to $r s$. In view of the need to ensure that the parameters still conform to the basic sequence and probability distribution feature settings, the study is carried out by controlling EDPs' cultural risk. The corresponding continuous node variables are prelearned for network inference in the form of a set. The essence of this learning process is the same feature set of discrete Bayesian network parameters, in order to facilitate risk control efficiency under different investment risk control economic benefits. Unified conversion and conversion of EDPs' cultural risk control benefits to varying degrees through various discrete processes:

$$
r s\left(\theta s \mid r s^{h}, \psi\right)=\prod_{i=1}^{n} \prod_{j=1}^{q_{i}} r s\left(\theta_{i j} \mid r s^{h}, \psi\right) .
$$

According to the prior probability corresponding to formula (20), combined with formula (22), the posterior probability based on formula (21) can be obtained, and the corresponding random variable probability conversion is performed:

$$
R S_{i}=\left(\begin{array}{c}
r s_{i 1} \\
r s_{i 2} \\
\cdots \\
r s_{i T}
\end{array}\right)=\left(\begin{array}{ccccc}
r s_{i, 0} & r s_{i, 11} & r s_{i, 12} & \ldots & r s_{i, 1 k} \\
r s_{i, 1} & r s_{i, 21} & r s_{i, 22} & \ldots & r s_{i, 2 k} \\
\cdots & \cdots & \cdots & \cdots & \cdots \\
r s_{i, T-1} & r s_{i, T 1} & r s_{i, T 2} & \cdots & r s_{i, T k}
\end{array}\right)\left(\begin{array}{c}
V_{i} \\
k_{i 1} \\
\ldots \\
k_{i k}
\end{array}\right)+\left(\begin{array}{c}
X_{i 1} \\
X_{i 2} \\
\ldots \\
X_{i T}
\end{array}\right) .
$$

Therefore, by including the total number of variables with different parameter indexes of $V$ and $\kappa$, the dynamic section data information based on Bayesian network decision reasoning can be obtained through different sections, that is, according to the prior probability, posterior probability, and its average. The processing method is distributed to the normal control to obtain a cross-section data oriented to the EDPs' cultural risk control, then the posterior probability is obtained according to different decision-making economies.

Finally, according to different degrees of EDPs' cultural risk control economic benefits, the prior probabilities, posterior probabilities, and corresponding conditional probability distributions of the paged network learning conditions are progressively advanced layer by layer [26]. Among them, the firstorder prior probability is obtained, the investment risk control decision-making conditions are based on the high control effect, medium control effect, and low control effect, and the corresponding risk control levels are, respectively, the low-risk level, the medium-risk level, and the high-risk level according to the degree of investment liabilities.

\section{Importance of the Proposed Policy}

7.1. Proposed Fault Tree Analysis. We have proposed corresponding policies to address the issues of human rights protection and the risk of cultural loss encountered by EDPs. For our proposed policy, we used fault tree analysis to statutory and quantitatively verify the effect of the policy after implementation to determine its importance. Taking policy recommendations as the bottom event and risk resolution as the top event, the probability of the top event is calculated to analyze the importance of the policy.

\subsection{Establishment of Fault Tree Model}

7.2.1. Identify Relevant Elements. We take EDPs' human rights guarantee and cultural protection as top events. The next level regards the protection of human rights and cultural protection as an intermediate event. Intermediate events are in the middle of the top and bottom events. It is also a classification of top events. We assign policies corresponding to the protection of human rights and cultural protection to the bottom layer of the two intermediate events. A fault tree model is formed by connecting the AND gate or the OR gate.

7.2.2. Fault Tree Flowchart. During the establishment of the fault tree, the top-level events are linked to the bottom-level events through the logic gates of the OR gate and the AND 
gate. In the protection of human rights and culture of EDPs, the influence of policy as the bottom event is regulated to the corresponding intermediate event [27]. The process is as shown in Figure 4 and Table 3.

7.2.3. Fault Tree Quantitative Analysis. The quantitative analysis of the fault tree refers to calculating the probability of occurrence of the top event of the system and some reliability indicators of the system. The probability of the top event is obtained from the probability of the bottom event. We change the policy to observe the probability change of the top event and then evaluate the importance of the policy [28].

Before the analysis, we need to introduce the definition of cut sets. A cut set is a set of basic times that causes a top event to occur. For a top event $T$ containing $n$ minimum cut sets, the probability of the top event occurring is as shown in formula (24).

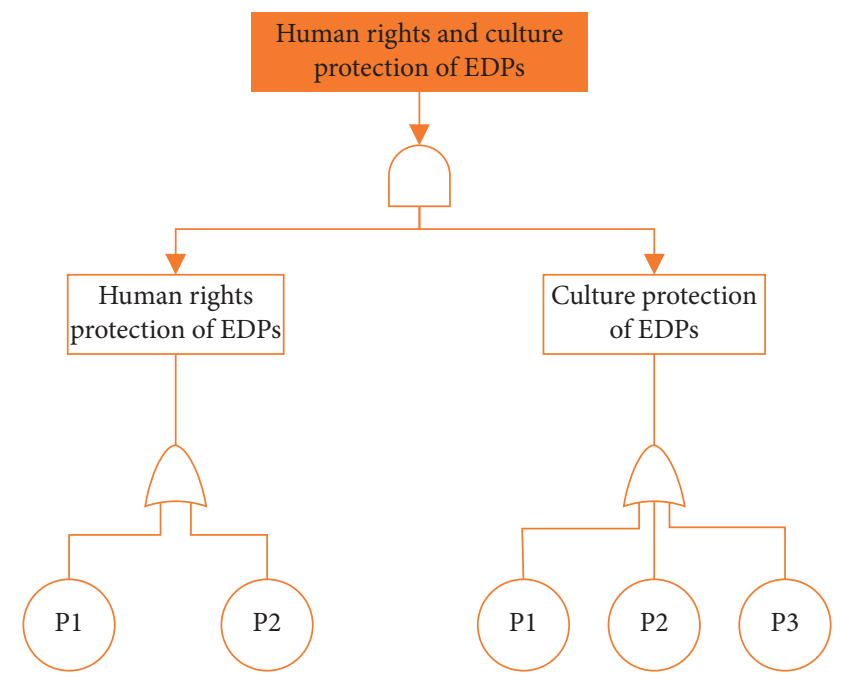

FIgURE 4: Fault tree model.

$$
P T=\sum_{i=1}^{n} P(c i)-\sum_{i=1}^{n-1} \sum_{j=i+1}^{n} P(c i c j)+\sum_{i=1}^{n-2} \sum_{j=i+1}^{n-1} \sum_{k=j+1}^{n}\left[P\left(c i c j c k+\cdots+(-1)^{n} P(c 1 c 2 c n)\right)\right] .
$$

When the number of minimum cut sets is large enough, the probability of top event in practical engineering calculation can be approximately as shown in formula (25).

$$
P_{T}=\sum_{i=1}^{n} P\left(c_{i}\right)
$$

The importance degree is a scale for evaluating the influence of each bottom event in the fault tree on the top event of the system. The formula for calculating the importance of the bottom event $X_{i}$ is as shown in formula (26).

$$
P_{X_{i} \mid T}=\frac{P x_{i}}{P_{T}},
$$

where $P_{X_{i}}$ indicates the probability of the bottom event occurring and $P_{T}$ indicates the probability of the top event $T$ occurring $[29,30]$.

The importance of all the basic events is ranked from large to small, and the importance of the basic events is ranked. The importance of the proposed policy can be analyzed by ranking.

7.3. Policy Implementation Effects. Define the ratio of the population with cultural background to the total population as the population of cultural species. When the culture goes extinct, the ratio becomes 0 . The maximum value of the ratio is $100 \%$. Factors such as the population, language, and geography of a country constrain the development of culture, which can be regarded as resources required by culture. When a foreign culture enters a country, it will compete with the native culture for the same resources such as the population that recognizes the culture, like species invasion.

$$
\left\{\begin{array}{l}
\frac{\mathrm{d} N_{1}}{\mathrm{~d} t}=N_{1}\left(1-\frac{N_{1}}{K_{1}}-\alpha_{21} \frac{N_{2}}{K_{1}}\right), \\
\frac{\mathrm{d} N_{2}}{\mathrm{~d} t}=N_{2}\left(1-\frac{N_{2}}{K_{2}}-\alpha_{12} \frac{N_{1}}{K_{2}}\right) .
\end{array}\right.
$$

Take Canada as an example to study the competition between local culture and culture of EDP. In the model, the culture of EDP is foreign culture and Canadian culture is native culture. Using a logistic curve to fit the Canadian population over a period of 2000 to 2019, the Canadian population in 2070 is predicted to be $3.8 \times 10^{7}$. So, the initial value of $N_{1}$ is equal to $92.7 \%$, and that of $N_{2}$ is equal to $7.3 \%$. Without interference, the native culture has a stronger ability to adapt to the local environment and use resources, so it has an advantage in cultural competition. In this condition, $0<\alpha_{21}<\alpha_{12}$. The result is shown in Figure 5.

If the policy to protect the EDPs' culture is implemented, the competitiveness of EDPs' culture in cultural competition will increase. In this condition, $0<\alpha_{21}, 0<\alpha_{12}$, and $\alpha_{21} \approx \alpha_{12}$. The result is shown in Figure 6 .

Contrasting Figure 5 with Figure 6, the implementation of cultural protection policy makes the existence of EDPs' culture longer.

\section{Conclusions}

In response to the issues of human rights protection and cultural preservation of EDPs, our first policy is to clarify EDPs' coverage. The implementation of this policy predicts island disasters caused by climate change on the one hand and implements rescue measures for high-risk island states in EDPs in advance, reducing material resources lost due to 
TABLE 3: Explanation of symbols.

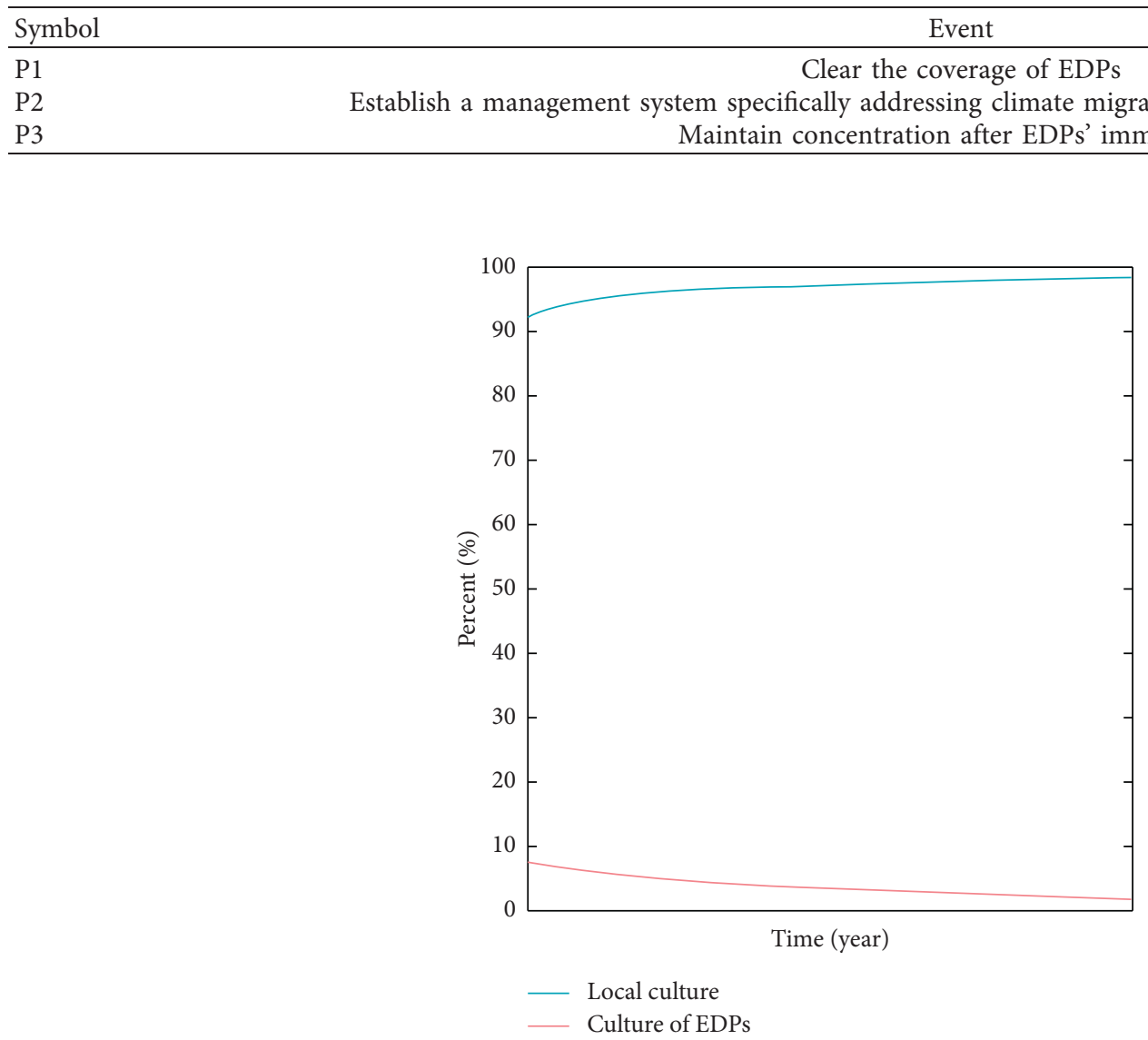

Figure 5: Population of culture over time without interference.

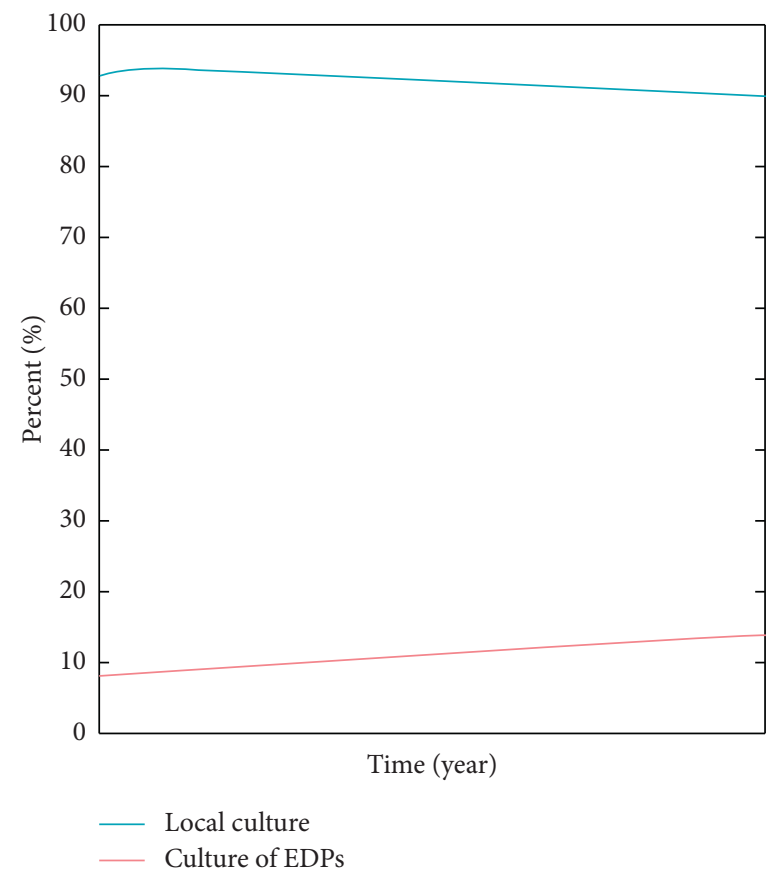

Figure 6: Population of culture over time in the case of policy implementation. 
emergency; on the other hand, it can carry out immigration preparation activities in advance and communicate with EDPs in advance [31]. Consult with neighboring countries of high-risk island countries, prepare in advance by receiving countries, and enact adaptation policies to reduce economic losses and social stress caused by large-scale migration. Contact climate experts to regularly analyze the coverage of EDP, predict the development of nearby climate, and prevent natural disasters such as typhoons and tsunamis. Such a coverage mechanism also contributes to the study of world geography. At the same time, migration also helps to protect the preservation and transmission of EDPs' cultures. Compared with remote immigrant cultures, receiving countries with similar living environments to immigrants are more likely to be recognized by the new living environment.

The second policy is to promulgate relevant systems to address climate migration, continuously improve the relevant system in accordance with the actual situation, and ensure the protection of EDPs' human rights to the greatest extent under other circumstances with the least impact. At the same time, related systems and early warning mechanisms were linked to form not only the relief work but also the prevention, reducing losses, and saving more climate refugees and natural resources $[32,33]$ and at the same time establish and improve the EDPs' immigration management system and comprehensively protect EDPs from the aspects of culture and human rights. Under the EDPs' immigration management system, relevant departments adopt various cultural preservation measures to reduce the disappearance of EDPs' culture and develop excellent culture and unique technologies of EDPs and set up a set of supervision and punishment measures to treat EDPs unfairly, without giving EDPs corresponding human rights penalties and corrections [34].

We have also proposed a policy to maintain the concentration of EDPs' migration, which is proposed for cultural protection. Decentralized migration of EDPs and migration to different countries are only a small part. Under the strong cultural system of the original inhabitants, cultural loss and cultural assimilation are prone to occur. Therefore, by adopting the method of maintaining concentration, we can better preserve the culture and preserve the original civilization.

\section{Data Availability}

The data used to support the findings of this study are included within the article.

\section{Conflicts of Interest}

The authors declare that there are no conflicts of interest regarding the publication of this paper.

\section{Acknowledgments}

This study was funded by the Humanities and Social Sciences Research Major Project of the Education Department of
Anhui Province (SK2017A0452), the Provincial Natural Science Foundation of Anhui (1808085MC88), and Key Project of Research Innovation Fund for Students of Anhui University of Finance and Economics (XSKY2107ZD).

\section{References}

[1] M. Toni and M. S. Marina, "Environmentally displaced persons," Environmental Policy and Law, vol. 48, no. 2, pp. 133-137, 2018.

[2] A. Lopez, "The protection of environmentally-displaced persons in international law," Environmental Law, vol. 37, no. 2, pp. 365-409, 2007.

[3] B. Havard, "Seeking protection: recognition of environmentally displaced persons under international human rights law," Villanova Environmental Law Journal, vol. 1, p. 65, 2007.

[4] S. Hayat, S. Wang, and J.-B. Liu, "Valency-based topological descriptors of chemical networks and their applications," Applied Mathematical Modelling, vol. 60, pp. 164-178, 2018.

[5] A. H. Westing, "Environmental refugees: a growing category of displaced persons," Environmental Conservation, vol. 19, no. 3, pp. 201-207, 1992.

[6] E. Naser-Hall, "Square pegs in round holes: the case of environmentally displaced persons and the need for a specific protection regime in the United States," Tulane Journal of International \& Comparative Law, 2014.

[7] M.-J. Shi, "The application of ARIMA model in investing forecast in fixed assets of Shanghai," Application of Stats and Management, 2005.

[8] P. Newbold, "ARIMA model building and the time series analysis approach to forecasting," Journal of Forecasting, vol. 2, no. 1, pp. 23-35, 2010.

[9] S. Voronin and J. Partanen, "Forecasting electricity price and demand using a hybrid approach based on wavelet transform, ARIMA and neural networks," International Journal of Energy Research, vol. 38, no. 5, pp. 626-637, 2014.

[10] J. Clifton and C. Majors, "Culture, conservation, and conflict: perspectives on marine protection among the bajau of southeast asia," Society \& Natural Resources, vol. 25, no. 7, pp. 716-725, 2012.

[11] A. Arnör, No State, No Protection, Exploring the Legal Protection for Environmentally Displaced Persons, Lund University, Lund, Sweden, 2013.

[12] M. F. M. Deng, "Report on the human rights of internally displaced persons on the profiles in displacement: east timor," Investment Analysts Journal, vol. 23, no. 8, pp. 45-59, 1997.

[13] C. Ariton-Gelan, "Policies of preservation and protection of the culture and identity of immigrants," International Conference Knowledge-Based Organization, vol. 21, no. 2, pp. 392-397, 2015.

[14] E. S. Vrnceanu, "European practices for the integration of immigrants," Revista De Stiinte Politice, 2015.

[15] J.-B. Liu, J. Zhao, H. He, and Z. Shao, "Valency-based topological descriptors and structural property of the generalized sierpiński networks," Journal of Statistical Physics, vol. 177, no. 6, pp. 1131-1147, 2019.

[16] I. Luljeta, "Human rights of irregular immigrants: a challenge for the universality of human rights," Academicus International Entific Journal, 2013.

[17] K. K. Moberg, "Extending refugee definitions to cover environmentally displaced Persons displaces necessary protection," Iowa Law Review, 2009.

[18] B. Mccormick, G. H. Hanson, and T. Boeri, Immigration Policy and the Welfare System: A Report for the Fondazione 
Rodolfo Debenedetti, Oxford University Press, Oxford, UK, 2002.

[19] J.-B. Liu, J. Zhao, and J. Min, "on the Hosoya index of graphs formed by a fractal graph," Fractals-Complex Geometry Patterns and Scaling, vol. 27, no. 8, 2019.

[20] Y. Hristov, "Social assessment of policies for labour, educational and cultural integration of immigrants in bulgaria," Nauchni trudove, 2015.

[21] J. Bernard and R. Mikešová, "The socio-cultural integration of immigrants: in between temporary migration and permanent settlement," Czech Sociological Review, vol. 50, no. 4, pp. 521-546, 2014.

[22] C. John and S. J. Reed, "The cultural integration of immigrants," American Catholic Sociological Review, vol. 21, no. 1, p. 89, 1959.

[23] M. P. Lindo, Structural and Socio-Cultural Integration of Immigrants in Welfare States, 2003.

[24] Y. Algan, A. Bisin, and T. Verdier, "Perspectives on Cultural Integration of Immigrants," PSE-Ecole d'économie de Paris (Postprint), 2012.

[25] N. Saba, "The protection of environmentally displaced persons and their right to a healthy and sustainable environment," International Journal of Private Law, vol. 2, no. 1, p. 76, 2009.

[26] Y. W. Chen and Y. F. Gao, "Overview of Bayesian network extension research," Control and Decision, vol. 23, no. 10, pp. 1081-1086, 2008.

[27] J. Ching and S.-S. Leu, "Bayesian updating of reliability of civil infrastructure facilities based on condition-state data and fault-tree model," Reliability Engineering \& System Safety, vol. 94, no. 12, pp. 1962-1974, 2009.

[28] P. Bucci, J. Kirschenbaum, L. A. Mangan, T. Aldemir, C. Smith, and T. Wood, "Construction of event-tree/fault-tree models from a Markov approach to dynamic system reliability," Reliability Engineering \& System Safety, vol. 93, no. 11, pp. 1616-1627, 2008.

[29] D. Heckerman, D. Geiger, and D. M. Chickering, "Learning bayesian networks: the combination of knowledge and statistical data," Machine Learning, vol. 20, no. 3, pp. 197-243, 1995.

[30] D. Heckerman, "Bayesian networks for data mining," Data Mining and Knowledge Discovery, vol. 1, no. 1, pp. 79-119, 1997.

[31] M. F. N. Franke, "The displacement of the rights of displaced persons: an irreconciliation of human rights between place and movement," Journal of Human Rights, vol. 7, no. 3, pp. 262-281, 2008 .

[32] J.-M. Zhu, L. Wang, and J.-B. Liu, "Eradication of Ebola based on dynamic programming," Computational and Mathematical Methods in Medicine, vol. 2016, Article ID 1580917, 9 pages, 2016.

[33] N. Friedman, M. Linial, and I. Nachman, "Using bayesian networks to analyze expression data," Journal of Computational Biology, vol. 7, no. 3-4, pp. 601-620, 2000.

[34] S. Verron, J. Li, and T. Tiplica, "Fault detection and isolation of faults in a multivariate process with Bayesian network," Journal of Process Control, vol. 20, no. 8, pp. 902-911, 2010. 